\title{
Torque Sensorless Control for an Electric Power Assisted Bicycle with Instantaneous Pedaling Torque Estimation
}

\author{
Takumi Kurosawa* ${ }^{*}$ Student Member, $\quad$ Yasutaka Fujimoto ${ }^{* a)}$ Senior Member
}

(Manuscript received May 2, 2016, revised Nov. 10, 2016)

\begin{abstract}
A typical electric power-assisted bicycle is equipped with an expensive torque sensor to measure a rider's pedaling torque. In this paper, we propose a method to estimate the pedaling torque from Hall effect sensors mounted on the motor, reducing the cost of electric power-assisted bicycles. It is necessary to separate pedaling torque and load torque from disturbance torque, which is estimated by a disturbance observer. We employ the Fourier series expansion to extract the periodic components of disturbance torque associated with the crank angle, which corresponds to the average pedaling torque. A conditional recursive least squares method is then used to estimate the parameters of the load torque model. Finally, the instantaneous pedaling torque is obtained based on the disturbance observer and the estimated load torque. We confirmed the validity of the proposed method by simulations and experiments. Our method can also be applied to a normal bicycle by replacing its front wheel with a motorized wheel.
\end{abstract}

Keywords: electric power assisted bicycle, Recursive least squares method, disturbance observer, pedaling torque estimator

\section{Introduction}

In recent years, environmentally-friendly devices have attracted many people. Electric power assisted bicycle and electric motorcycle are one of them because they do not exhaust $\mathrm{CO}_{2}$. Many companies have released a lot of models of electric power assisted bicycles and electric motorcycles. Their sales have been increasing compared to motorcycle's. There are a lot of studies which focus on development and control of electric bicycles. Defoort and Murakami developed an autonomous bicycle which can keep own posture by sliding-mode control ${ }^{(1)}$. They analyzed and desinged a robust controller for the autonomous bicycle. Hsu et al. proposed assist power management by reinforcement-learning ${ }^{(2)}$. They achieved better energy utilization satisfying requirement of quality of ride. Chen et al. proposed a velocity control approach for an electric bike ${ }^{(3)}$. They focused on two types of torque (velocity-dependent torque and deviation torque) in order to realize velocity control.

There are a lot of different points between electric power assisted bicycles and electric motorcycles. Necessity of the license is the one of them. The license is necessary in order to use electric motorcycles. On the other hand, most of the people can use electric power assisted bicycles because license is unnecessary. Bicycles are driven by pedaling torque (human power) and electric power. In particular in Japan, assisted power of the electric motor depends on pedaling torque and bicycle's speed, which is specified by the Japanese Road Traffic Law. In order to measure the pedaling torque, an expensive torque sensor is equipped in the crank box of the

\footnotetext{
a) Correspondence to: Yasutaka Fujimoto. E-mail: fujimotoyasutaka-by@ynu.ac.jp

* Yokohama National University

79-5, Tokiwadai, Hodogaya-ku, Yokohama, Kanagawa 2408501, Japan
}

bicycle. If the pedaling torque is estimated without such an expensive torque sensor, it can be removed from the bicycle. Therefore, the purpose of this study is to estimate the pedaling torque, moreover the final goal of our research is development of a wheel-type-assist kit including a control unit. We consider a front-wheel assisted bicycle because the frontwheel of non-assisted bicycles is easy to be replaced with the assist wheel kit.

There is a research on a pedaling torque estimation for a two-wheel-drive electric bicycle. Kawajiri et al. proposed an estimation method by using information of the front and rear wheels ${ }^{(4)}$. This method needs two rotary encoders and an accelerometer.

In this study, we proposed a pedaling torque estimator to estimate pedaling torque ${ }^{(5)(6)}$. This estimator only uses hole sensor information of a single blushless motor. However, estimation accuracy was not sufficient to realize comfortable assistance. In this paper, we propose an estimation method of the pedaling torque by the recursive least squares method with update condition. Thanks to the update condition, estimation accuracy can be improved higher than the conventional method.

The configuration of this paper is shown below. A model of the electric power assisted bicycle of front wheel assisttype is derived in Section 2. The estimation method of the pedaling torque is described in Section 3. In order to show the validity of the proposed method, simulations and experimental results are shown in Section 4 and Section 5. Finally, this paper is concluded in Section 6.

\section{Electric Power-Assisted Bicycle}

In this Section, we introduce a model of a front-wheelassisted bicycle. A motor can be easily installed into the front wheel compared with the rear wheel because the rear wheel is connected to the pedals via chain. Figure 1 shows 


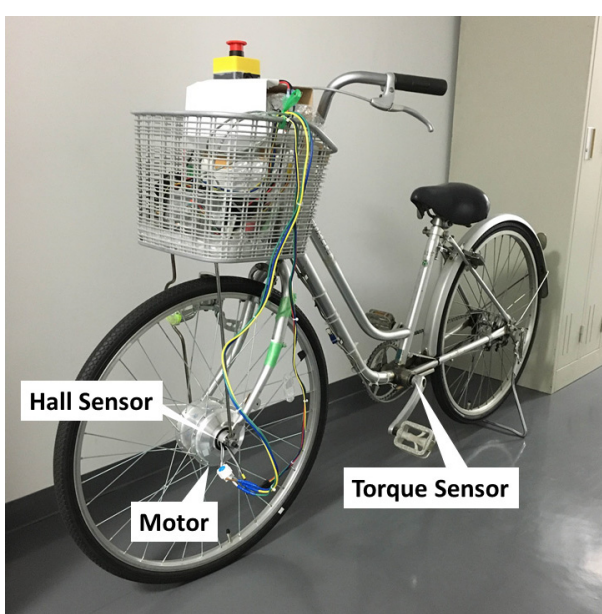

Fig. 1. Electric power assisted bicycle

Table 1. Parameters of electric power-assisted bicycle

\begin{tabular}{cl}
\hline \hline$V_{q}$ & q axis voltage of armature winding \\
\hline$I_{q}$ & q axis current of armature winding \\
\hline$R$ & Resistance of armature winding \\
\hline$K_{e}$ & Back-EMF constant \\
\hline$K_{\tau}$ & Torque constant \\
\hline$\tau_{\text {roll }}$ & Rolling torqu \\
\hline$\tau_{\text {inc }}$ & Inclined road toque \\
\hline$\tau_{\text {vis }}$ & Viscous friction torque \\
\hline$\tau_{\text {air }}$ & Aerodynamic drag torque \\
\hline$J$ & Inertia of rotor \\
\hline$J_{n}$ & Nominal value of inertia of rotor \\
\hline$M$ & Mass of bicycle and rider \\
\hline$r$ & Wheel radius \\
\hline$v$ & Velocity of bicycle \\
\hline$\omega$ & Angular velocity of wheel \\
\hline$F_{\text {motor }}$ & Motor force \\
\hline$F_{\text {man }}$ & Pedaling force \\
\hline$F_{\text {load }}$ & Load force \\
\hline$\tau_{\text {motor }}$ & Motor torque \\
\hline$\tau_{\text {man }}$ & Pedaling torque \\
\hline$\tau_{\text {load }}$ & Load torque \\
\hline$\theta_{c}$ & Angle of crank \\
\hline$G$ & Gear ratio \\
\hline \hline
\end{tabular}

the experimental system of the front-wheel-assisted bicycle. The assist torque is determined from the information of the pedaling torque and the speed of the bicycle. The motion equation of the bicycle is given as follows:

$$
M \dot{v}=F_{\text {motor }}+F_{\text {man }}-F_{\text {load }}
$$

Parameters appeared in the equation are denoted in Table 1. Equation (1) can be equivalently transformed into the motion equation around the front wheel axis as follows:

$$
J \dot{\omega}=\tau_{\text {motor }}+\tau_{\text {man }}-\tau_{\text {load }}
$$

where $J=M r^{2}, \tau_{\text {motor }}=r F_{\text {motor }}, \tau_{\text {man }}=r F_{\text {man }}, \tau_{\text {load }}=$ $r F_{\text {load }}$, respectively. If we assume that the electrical time constant of the motor is short enough and the motor is not salient, q-axis current $I_{q}$ and the motor torque $\tau_{\text {motor }}$ are described by

$$
\begin{aligned}
& I_{q}=\frac{1}{R}\left(V_{q}-K_{e} \omega\right) \\
& \tau_{\text {motor }}=K_{\tau} I_{q} \ldots \ldots
\end{aligned}
$$

Eventually, the plant model(2) is rewritten as follows:

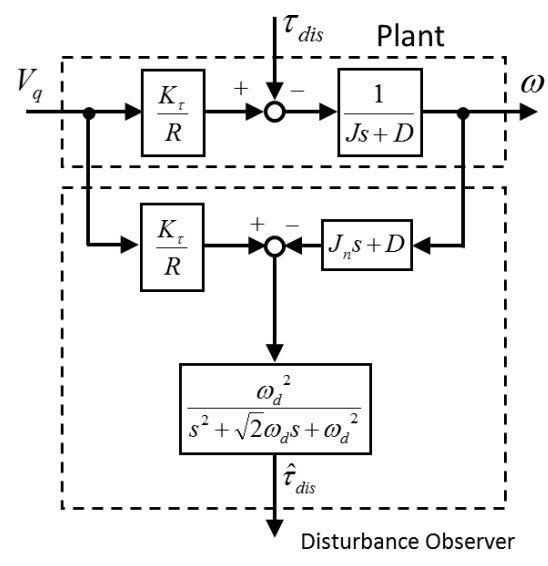

Fig. 2. Model of disturbance observer

$$
J \dot{\omega}=\frac{K_{\tau}}{R} V_{q}-D \omega-\tau_{d i}
$$

where $D=K_{\tau} K_{e} / R . \tau_{d i s}$ is the total disturbance torque defined as follows:

$$
\tau_{\text {dis }}=\tau_{\text {load }}-\tau_{\text {man }}
$$

In this study, we assume that the disturbance torque consists of the load torque and the pedaling torque.

\section{Pedaling Torque Estimator (PTE)}

This section describes how to estimate the pedaling torque. From Eq. (6), the estimated pedaling torque is calculated by as follows:

$$
\hat{\tau}_{\text {man }}(t)=\hat{\tau}_{\text {load }}(t)-\hat{\tau}_{\text {dis }}(t)
$$

where $\hat{\tau}_{\text {load }}, \hat{\tau}_{\text {dis }}$ are estimated load torque and estimated disturbance torque, respectively. These values have to be obtained to estimate the pedaling torque.

3.1 Disturbance Observer (DOB) First, we utilize the disturbance observer in order to estimate the disturbance torque $^{(7)}$. In our experimental system, Hall effect sensors are attached at the motor to measure electric angle of rotor. By using these sensors, velocity of the bicycle is calculated. We utilize a time counter method to calculate the velocity accurately ${ }^{(8)}$. However, the observation noise is obvious because the angle resolution of the wheel is 0.013rad. In order to decrease the observation noise, we utilize a second-order lowpass filter (LPF) in the DOB. A second-order Butterworth LPF is adopted for this purpose. The disturbance torque applied to the front wheel axle can be estimated as follows:

$$
\hat{\tau}_{d i s}(t)=\frac{\omega_{d}^{2}}{s^{2}+\sqrt{2} \omega_{d} s+\omega_{d}^{2}}\left(\frac{K_{\tau}}{R} V_{q}-D \omega-J_{n} s \omega\right)
$$

where $\omega_{d}$ is the cut-off frequency of the disturbance observer. Figure 2 shows the block diagram of the second-order disturbance observer.

3.2 Load Torque Estimator Second, we describe how to estimate the load torque. A load torque estimator focuses on the periodic characteristics of the pedaling motion. In this study, we calculate the average pedaling torque and the average load torque by using information of the preceding crank cycle. The average value is not an instantaneous 
Table 2. Parameter of load torque

\begin{tabular}{lll}
\hline \hline$\mu$ & Coefficient of rolling friction resistance & 0.007 \\
\hline$g\left[\mathrm{~m} / s^{2}\right]$ & Acceleration of gravity & 9.8 \\
\hline$\delta[\mathrm{rad}]$ & Angle of inclination & 0 \\
\hline$D_{M}\left[\mathrm{Nm} \mathrm{rad}^{-1} \mathrm{~s}^{-1}\right]$ & Viscosity friction coefficient & 3.00 \\
\hline$\rho\left[\mathrm{N} \mathrm{s}^{2} \mathrm{~m}^{-4}\right]$ & Atmospheric density & 1.208 \\
\hline$A\left[\mathrm{~m}^{2}\right]$ & Frontal projected area & 0.67 \\
\hline$C_{d}$ & Air resistance coefficient & 0.9 \\
\hline \hline
\end{tabular}

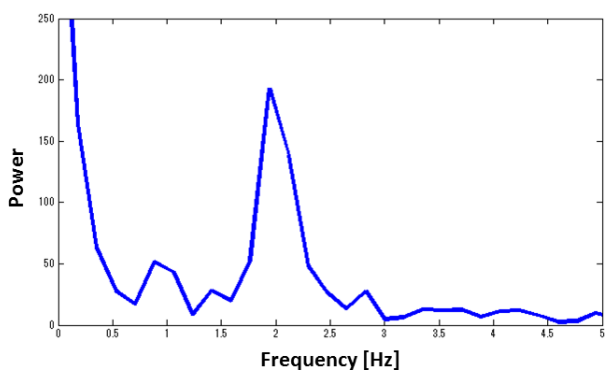

Fig. 3. The FFT result of the actual pedaling torque

value, but we assume that the load torque does not change suddenly. Then, in order to estimate the instantaneous load torque, we adopt the recursive least squares method (RLS). In this study, we assume that the load torque contains several components as follows ${ }^{(9)}$ :

$$
\begin{array}{r}
\tau_{\text {load }}=\mu M g r+M g r \sin \delta+D_{M} \omega+\frac{1}{2} \rho A C_{d} r^{3} \omega^{2} \\
\ldots \ldots \ldots \ldots \ldots \ldots \ldots \ldots \ldots \ldots \ldots
\end{array}
$$

where, $\tau_{\text {roll }}, \tau_{\text {inc }}, \tau_{\text {vis }}, \tau_{\text {air }}$ are rolling torque and inclined road torque, viscous friction torque, and aerodynamic drag torque, respectively. Parameters of load torque are shown in Table 2. If we focus on the angular velocity in Eq. (9), the load torque can be divided into three terms as follows: an independent term on the angular velocity, a term proportional to the angular velocity, and a term on quadratically proportional to the angular velocity.

$$
\tau_{\text {load }}=K_{0}+K_{1} \omega+K_{2} \omega^{2} \cdots
$$

From the Eq. (11), the RLS method can identify the parameters $K_{0}, K_{1}, K_{2}$ if we obtain the value of $\tau_{\text {load }}$ and $\omega$. In Section 3.2.1, we describe how to substitute average value $\tilde{\tau}_{\text {load }}$ for unknown value $\tau_{\text {load }}$. In Section 3.2.2, we apply the RMS method in order to estimate the instantaneous load torque.

3.2.1 Average Load Torque Estimator In order to apply RLS method to Eq. (11), we have to obtain the value of $\tau_{\text {load }}$. Here we focus on the periodic characteristic of the pedaling motion to substitute the average value for the unknown value. Figure 3 shows the FFT result of an actual pedaling torque measured by a torque sensor. The measurement was conducted when we ride the bicycle on a flat road. The horizontal axis represents the frequency of the cycle of the crank. From Fig. 3, we can confirm that the pedaling torque has not only the second harmonic frequency but also the fundamental frequency with respect to the crank angle. Thanks to Fourier series expansion, we can pick out these components. Therefore, we reconstruct the average pedaling torque from these components by information of preceding crank cycle.

$$
\begin{aligned}
& a_{i}=\frac{1}{\pi} \int_{0}^{2 \pi} \tilde{\tau}_{d i s}\left(\theta_{c}\right) \cos i \theta_{c} d \theta_{c} \\
& b_{i}=\frac{1}{\pi} \int_{0}^{2 \pi} \tilde{\tau}_{d i s}\left(\theta_{c}\right) \sin i \theta_{c} d \theta_{c}
\end{aligned}
$$

where, $\tilde{\tau}_{\text {dis }}$ is the esitmated disturbance torque with respect to the crank angle.

$$
\tilde{\tau}_{d i s}\left(\theta_{c}(t)\right)=\hat{\tau}_{d i s}(t)
$$

where $\theta_{c}$ is the crank angle which is obtained from the wheel angle $\theta_{\text {wheel }}$ as follows:

$$
\theta_{c}=G \theta_{\text {wheel }}
$$

where $G$ is the gear ratio between the rear wheel and the pedals connected by a chain. Then, the average pedaling torque with respect to the crank angle $\tilde{\tau}_{\text {man }}\left(\theta_{c}\right)$ can be reconstructed by

$$
\tilde{\tau}_{\text {man }}\left(\theta_{c}\right)=\sum_{i=1}^{2}\left(a_{i} \cos i \theta_{c}+b_{i} \sin i \theta_{c}\right)-C \cdots \cdots \cdots
$$

The term $C$ is a local minimum value so that $\tilde{\tau}_{\text {man }}\left(\theta_{c}\right) \geq 0$ satisfies. In order to calculate the local minimum value, a differential value of Eq. (16) by $\theta_{c}$ is needed. The differential value can be transformed to a quartic equation of $\sin \theta_{c}$. The quartic equation can be calculated by the Ferrari formula. $\tilde{\tau}_{\text {man }}\left(\theta_{c}\right)$ is composed of the fundamental components of the pedaling torque in the preceding crank cycle.

Finally, by using the average pedaling torque $\tilde{\tau}_{\text {man }}\left(\theta_{c}\right)$, we can obtain the average load torque $\tilde{\tau}_{\text {load }}\left(\theta_{c}\right)$ in preceding cycle as follows:

$$
\tilde{\tau}_{\text {load }}\left(\theta_{c}\right)=\tilde{\tau}_{d i s}\left(\theta_{c}\right)+\tilde{\tau}_{\text {man }}\left(\theta_{c}\right)
$$

We assume that the load torque does not change suddenly. $\tilde{\tau}_{\text {load }}\left(\theta_{c}\right)$ is substituted for $\tau_{\text {load }}$ in Eq. (11).

\subsubsection{Conditional Recursive Least Squares Method}

We apply the recursive least squares (RLS) method in order to estimate the instantaneous load torque. By using the average load torque $\tilde{\tau}_{\text {load }}\left(\theta_{c}\right)$ and the angular velocity $\omega$, RLS method can identify the coefficients $K_{0}, K_{1}, K_{2}$ by using equations as follows:

$$
\begin{gathered}
\boldsymbol{\Omega}(k)=\boldsymbol{\Omega}(k-1)-\boldsymbol{P}(k) \boldsymbol{\xi}(k)\left(\boldsymbol{\xi}^{T}(k) \boldsymbol{\Omega}(k-1)-y(k)\right) \\
\ldots \ldots \ldots \ldots \ldots \ldots \\
\boldsymbol{P}(k)=\frac{1}{\lambda}\left(\boldsymbol{P}(k-1)-\frac{\boldsymbol{P}(k-1) \boldsymbol{\xi}(k) \boldsymbol{\xi}^{T}(k) \boldsymbol{P}(k-1)}{\lambda+\boldsymbol{\xi}^{T}(k) \boldsymbol{P}(k-1) \boldsymbol{\xi}(k)}\right)
\end{gathered}
$$

where, an output can be expressed as $y=\boldsymbol{\xi} \boldsymbol{\Omega}$ by using an unknown parameter vector $\boldsymbol{\Omega}$ and an observable signal vector $\boldsymbol{\xi}$. Moreover, $\lambda$ is a forgetting factor which is slightly smaller than 1. In order to estimate the instantaneous load torque, $\boldsymbol{\Omega}, \boldsymbol{\xi}$, and $y$ are defined as follows:

$$
\begin{aligned}
& \boldsymbol{\Omega}:=\left[\begin{array}{lll}
\hat{K}_{0} & \hat{K}_{1} & \hat{K}_{2}
\end{array}\right]^{T} . \\
& \boldsymbol{\xi}:=\left[\begin{array}{lll}
1 & \omega & \omega^{2}
\end{array}\right] \ldots \ldots \\
& y:=\tilde{\tau}_{\text {load }}\left(\theta_{c}\right) . \\
& \ldots
\end{aligned}
$$




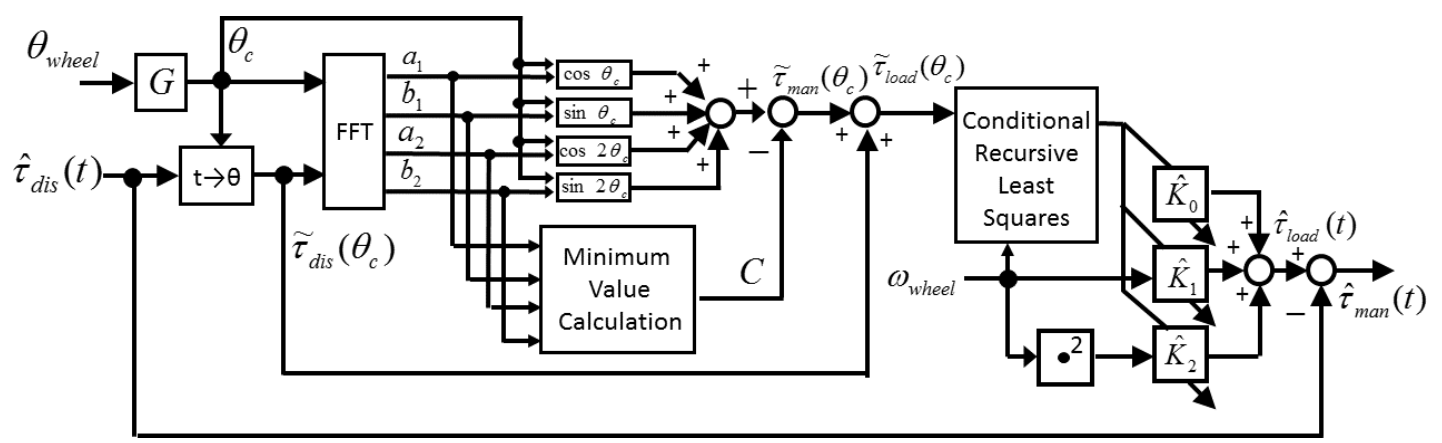

Fig. 4. The block diagram of PTE using conditional recursive least squares method

$$
\left[\begin{array}{l}
a_{1} \\
b_{1} \\
a_{2} \\
b_{2}
\end{array}\right]=\frac{1}{2 \pi}\left[\begin{array}{cccc}
\phi+\frac{\sin 2 \phi}{2} & \frac{1}{2}-\frac{\cos 2 \phi}{2} & 2 \sin \phi-\frac{4 \sin ^{3} \phi}{3} & \frac{4}{3}-\frac{4 \cos ^{3} \phi}{3} \\
\frac{1}{2}-\frac{4 \cos ^{3} \phi}{3} & \phi-\frac{\sin 2 \phi}{2} & 2 \cos \phi-\frac{4 \cos ^{3} \phi}{3}-\frac{2}{3} & \frac{4 \sin ^{3} \phi}{3} \\
2 \sin \phi-\frac{4 \sin ^{3} \phi}{3} & 2 \cos \phi-\frac{4 \cos ^{3} \phi}{3}-\frac{2}{3} & \phi+\frac{\sin 4 \phi}{4} & 1-\cos ^{4} \phi-\sin ^{4} \phi \\
\frac{4}{3}-\frac{4 \cos ^{3} \phi}{3} & \frac{4 \sin ^{3} \phi}{3} & 1-\cos ^{4} \phi-\sin ^{4} \phi & \phi-\frac{\sin 4 \phi}{4}
\end{array}\right]\left[\begin{array}{l}
A_{1} \\
B_{1} \\
A_{2} \\
B_{2}
\end{array}\right] \ldots
$$

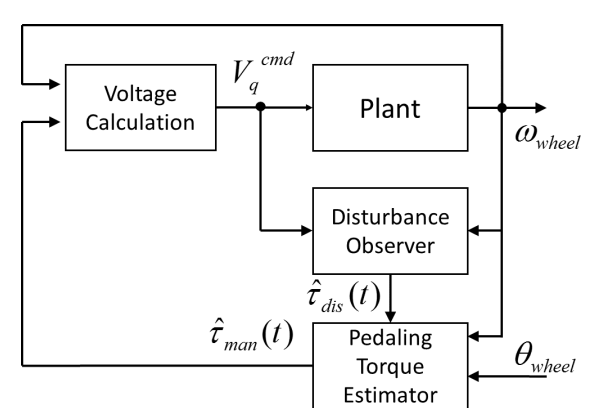

Fig. 5. Model of estimated pedaling torque

If the observable signal $\omega$ is small, the signal is not suitable signals to identify from the point of view of persistent excitation ${ }^{(10)}$. In this paper, if the angular velocity $\omega$ is smaller than $\boldsymbol{\epsilon}, \boldsymbol{\Omega}(k)$ and $\boldsymbol{P}(k)$ are not updated, i.e., $\boldsymbol{\Omega}(k)=\mathbf{\Omega}(k-1), \boldsymbol{P}(k)=$ $\boldsymbol{P}(k-1)^{(11)}$. The initial condition of $\boldsymbol{\Omega}$ is expressed as follows;

$$
\mathbf{\Omega}(0)=\left[\begin{array}{lll}
0 & 0 & 0
\end{array}\right]^{T}
$$

Figure 4 shows the block diagram of PTE using the conditional RLS method. The pedaling torque is estimated by the DOB and the PTE as shown in Fig. 5. In our system, the voltage command $V_{q}^{c m d}$ is determined by the estimated pedaling torque and the angular velocity (bicycle's speed). This estimator does not depend on the velocity of the bicycle because this system uses information of the crank angle and the angular velocity by the Hall effect sensor.

3.3 Initial Cycle of Crank As we explained above, PTE uses information of the preceding rotation of the crank. Therefore, there are not sufficient data, when the torque components are calculated in initial cycle of crank. An estimation error in initial one cycle of crank is caused by the lack of data. In this section, we utilize the correction coefficient in initial cycle of crank. Here, we assume that the pedaling torque consists as follows:

$$
\tau(\theta)=A_{1} \cos \theta+B_{1} \sin \theta+A_{2} \cos 2 \theta+B_{2} \sin 2 \theta .
$$

where $A_{1}, B_{1}, A_{2}, B_{2}$ are the torque components which have sufficient data. Then, each components can be calculated by

$$
\begin{aligned}
& a_{i}=\frac{1}{\pi} \int_{0}^{\phi} \tau\left(\theta_{c}\right) \cos i \theta_{c} d \theta_{c} \\
& b_{i}=\frac{1}{\pi} \int_{0}^{\phi} \tau\left(\theta_{c}\right) \sin i \theta_{c} d \theta_{c}
\end{aligned}
$$

where $\psi$ is an angle within initial cycle of $\operatorname{crank}(0<\phi<2 \pi)$. It is possible to rewrite from Eq. (26) and Eq. (27) as Eq. (18). If we set $\boldsymbol{\Omega}=\left[A_{1}, B_{1}, A_{2}, B_{2}\right]^{T}$ and $\boldsymbol{y}=\left[a_{1}, b_{1}, a_{2}, b_{2}\right]^{T}$, Eq. (18) can be considered as $\boldsymbol{y}=\boldsymbol{\xi} \boldsymbol{\Omega}$. Therefore, this equation can be calculated by RLS method expressed as Eq. (19) and Eq. (20). Here, we set the forgetting factor as 0.995. In the initial cycle of crank, the torque components are corrected by these equations.

\section{Simulation}

In this section we consider the validity of the proposed PTE by numerical simulations. The pedaling torque and the load torque are given as follows:

$$
\begin{aligned}
& \tau_{\text {man }}=\left\{\begin{array}{lll}
20\left(1+\cos 2 \theta_{c}\right) & \text { if } & t \leq 6 \\
30\left(1+\cos 2 \theta_{c}\right) & \text { if } \quad t>6
\end{array}\right. \\
& \tau_{\text {load }}=\left\{\begin{array}{l}
1.81+2.00 \omega+0.1 \omega^{2} \quad \text { if } \quad t \leq 4 \\
10.81+2.00 \omega+0.1 \omega^{2} \quad \text { if } \quad t>4
\end{array}\right.
\end{aligned}
$$

Table 3 shows simulation parameters we selected. The simulation results by the PTE using RLS method are expressed with Fig. 6. Compared with the conventinal results ${ }^{(12)}$ shown in Fig. 7, estimation accuracy is increasing. Thanks to the update condition and the correction in initial cycle of crank, the estimated pedaling torque is estimated more accurately. Especially, the estimation accuracy of the first pedaling motion is higher. When the load torque changed, RLS method can estimate the load torque. However, the estimation error causes the error of the pedaling torque estimation. In order to increase the estimation accuracy of the load torque, other 


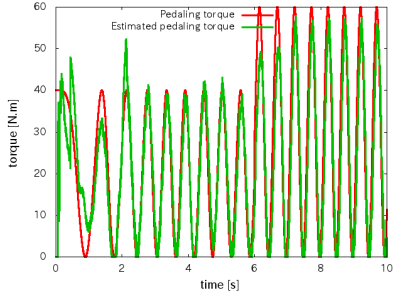

(a) Estimated pedaling torque.

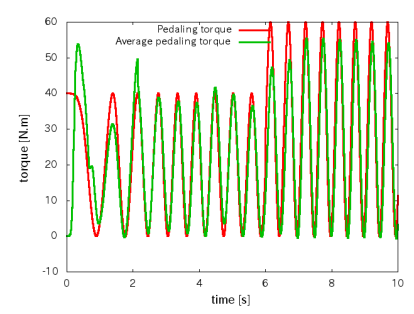

(b) Average pedaling torque

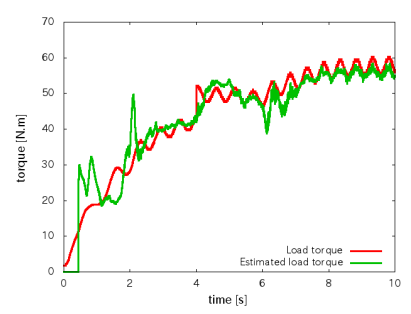

(c) Estimated load torque

Fig. 6. Simulation results. (proposed method)

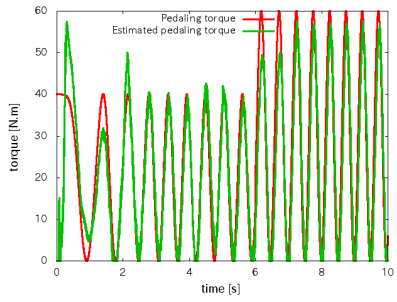

(a) Estimated pedaling torque.

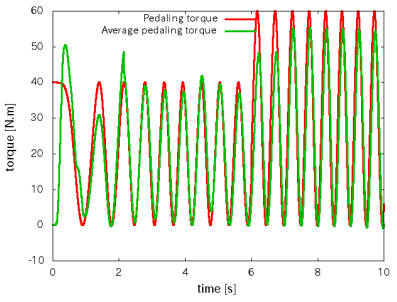

(b) Average pedaling torque.

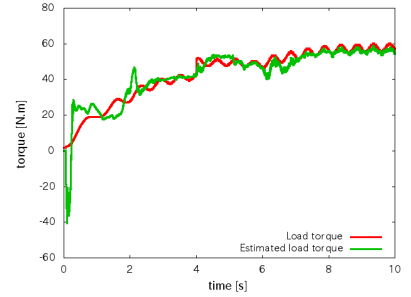

(c) Estimated load torque.

Fig. 7. Simulation results. (conventinal mehtod)

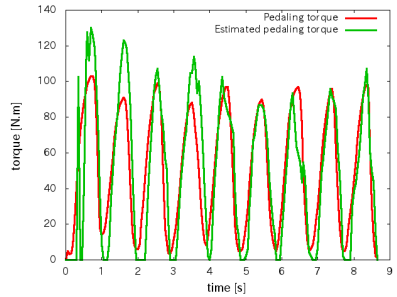

(a) Estimated pedaling torque.

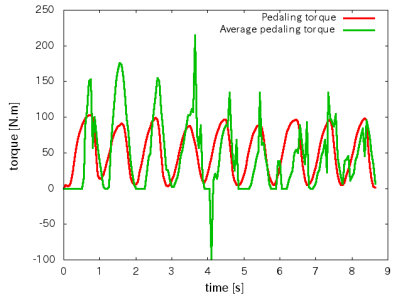

(b) Average pedaling torque.

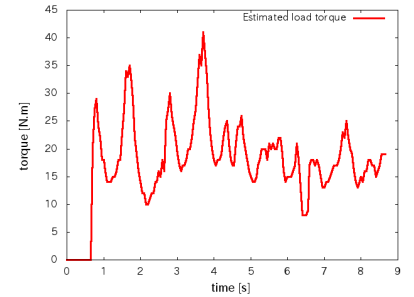

(c) Estimated load torque.

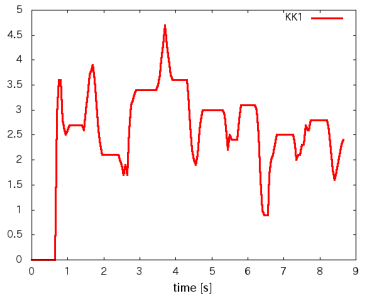

(d) Estimated parameter $K_{1}$

Fig. 8. Experimental results. (On-roller experiment without assistance)

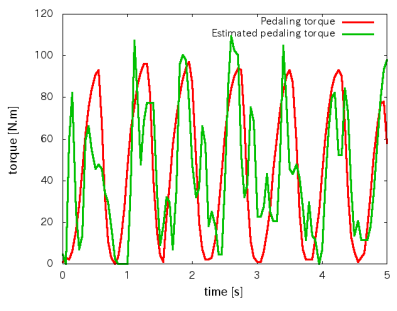

(a) Estimated pedaling torque.

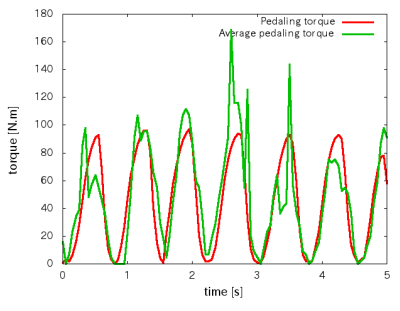

(b) Average pedaling torque.

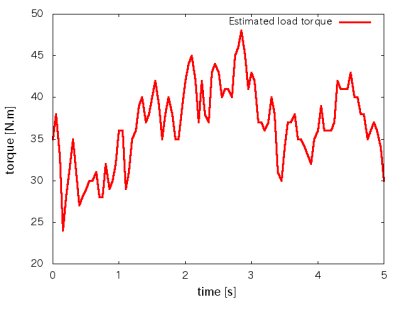

(c) Estimated load torque.

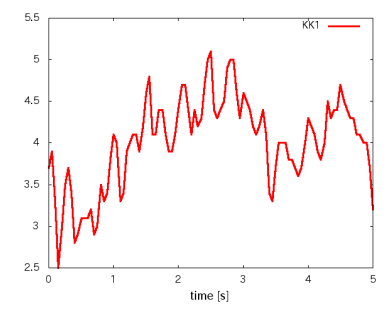

(d) Estimated parameter $K_{1}$.

Fig. 9. Experimental results. (On-road experiment with assistance by using the estimated pedaling torque)

Table 3. Simulation parameters

\begin{tabular}{lll}
\hline \hline$J_{n}\left[\mathrm{kgm} m^{2}\right]$ & Inertia of front wheel & 9.09 \\
\hline$D[\mathrm{Nms} / \mathrm{rad}]$ & Friction coefficient of front wheel & 3.00 \\
\hline$K_{\tau}[\mathrm{Nm} / \mathrm{A}]$ & Torque constant of motor & 0.708 \\
\hline$R[\Omega]$ & Resistance of armature winding & 0.20 \\
\hline$\omega_{d}[\mathrm{rad} / \mathrm{s}]$ & Cut-off frequency of DOB & 50 \\
\hline$\lambda$ & Forgetting factor & 0.999 \\
\hline \hline
\end{tabular}

method is needed. For instance, if an accelerometer is attached, a gradient of a road can be obtained. Figure 6(b) shows the average pedaling torque $\tilde{\tau}_{\text {man }}$, which has been reconstructed by the FFT computation. Here, we assume that the pedaling torque has periodic components. This assumption is used to decouple the pedaling torque and the load torque each other. We can observe that the average pedaling torque is well reconstructed from this figure. In Fig. 6(b), the average pedaling torque keeps the value over 0 thanks to the local minimum value.

\section{Experiment}

In this section, we investigate the validity of the proposed PTE by experiments. Our experimental system is shown in Fig. 1. The motor is brushless DC motor and the output is $250 \mathrm{~W}$. A torque sensor is equipped with in the crank box to compare the estimated pedaling torque and the measured pedaling torque. The experiments were conducted on a test roller and on a straight road without inclination. We set the experimental parameter as shown in Table 4. The experimental results are shown in Fig. 8 and Fig. 9. At first, in order to confirm the validity of the proposed method, we experiment on the test roller without assistance. From Fig. 8(a), the pedaling torque was almost estimated. In particular at first cycle of the crank, the estimation accuracy was higher than the conventional experiments. In Fig. 8(b), the average pedaling torque took negative value at $t=4$ [s]. In Eq. (16), the term $C$ is determined so that $\tilde{\tau}_{\text {man }} \geq 0$. In this experiment, 
Table 4. Experimental parameters

\begin{tabular}{lll}
\hline \hline$J_{n}\left[\mathrm{kgm} m^{2}\right]$ & Inertia of front wheel (Roller) & 1.50 \\
\hline$J_{n}\left[\mathrm{kgm} m^{2}\right]$ & Inertia of front wheel (Road) & 9.09 \\
\hline$D[\mathrm{Nms} / \mathrm{rad}]$ & Friction coefficient of front wheel & 0.275 \\
\hline$K_{\tau}[\mathrm{Nm} / \mathrm{A}]$ & Torque coefficient of motor & 0.708 \\
\hline$R[\Omega]$ & Resistance of armature winding & 0.20 \\
\hline$\omega_{d}[\mathrm{rad} / \mathrm{s}]$ & Cut-off frequency of DOB & 25 \\
\hline$\lambda$ & Forgetting factor & 0.995 \\
\hline \hline
\end{tabular}

the Newton method was used to find the solution instead of the Ferrari formula due to computational resource restriction, and it yielded a wrong solution at $t=4[\mathrm{~s}]$. From Fig. 8(c), the estimated load torque depends on the angular velocity by RLS method. Figure 8(d) shows an estimated parameter $K_{1}$ of the load torque. Since the forgetting factor has relatively small value which is 0.995 , the time constant of the convergence is relatively short which is about $0.2[\mathrm{~s}]$ with the sampling time 1 [ms]. The validity of the proposed method was confirmed by this experiment.

Next experiment was conducted on the road with assistance by the estimated pedaling torque. In spite of using the second-order LPF to decrease an observed signal, the estimated disturbance torque was oscillated. Therefore, the estimated load torque was oscillated. However, the torque components are obtained exactly from the result of the average pedaling torque. In order to give more comfort assistance, we applied a first-order LPF to the estimated pedaling torque. The result of the filtered pedaling torque is expressed by Fig. 9(a). The pedaling torque is estimated approximately, but there are oscillated points. The motor torque could be obtained by the estimated pedaling torque. As the future works, we have to decrease these oscillations. A fluent estimated pedaling torque contributes comfortable assistance.

\section{Conclusion}

This paper described the proposed PTE by RLS method with the update condition in order to increase the estimation accuracy. We confirmed increasing accuracy of the estimation by the simulation and the experiment. In particular on the test roller, the validity of the proposed method was confirmed. The pedaling torque was almost estimated on the test roller, but the oscillation was occurred on the road. As the future work, we must decrease the oscillation to obtain the comfortable assistance.

Authors would like to thank Mr. Takeji Tokumaru, E-bike Corporation, for his valuable comments and Mr. Norihito Fukushima, a graduate student of Yokohama National University, for his help.

\section{References}

( 1 ) M. Defoort and T. Murakami: "Sliding-Mode Control Scheme for an Intelligent Bicycle", IEEE Trans. Ind. Electron., Vol.56, No.9, pp.3357-3368 (2009)
( 2 ) R.C. Hsu, C. Liu, and D. Chan: "A Reinforcement-Learning-Based Assisted Power Management with QoR Provisioning for Human-Electric Hybrid Bicycle", IEEE Trans. Ind. Electron., Vol.59, No.8, pp.3350-3359 (2012)

( 3 ) P.C. Chen, C.C. Hsiao, S.L. Wu, and S.B. Chang: "The Torque Control with Multi-Objective Performance for E-Bike System with Human Power Assistance", WSEAS Trans. Systems and Control, Vol.6, No.10, pp.361-370 (2011)

( 4 ) H. Kawajiri, H. Mizoguchi, S. Sakaino, and T. Tsuji: "Sensorless Pedaling Torque Estimation by Front and Rear Wheels Independently Driven Power Assist Bicycle", Proc. IEEE ICM, pp.360-365 (2015)

( 5 ) T. Kurosawa, Y. Fujimoto, and T. Tokumaru: "Estimation of Pedaling Torque for Electric Power Assisted Bicycles", Proc. IEEE IECON, pp.2756-2761 (2014)

( 6 ) T. Kurosawa, Y. Fujimoto, and T. Tokumaru: "Estimation of Pedaling Torque for Electric Power-assisted Bicycle by Using Recursive Least Squares Method", Proc. IEEJ SAMCON, TT4-1-4 (2015)

( 7 ) K. Ohnishi: "Robust Motion Control by Disturbance Observer", Journal of Robotics and Mechatronics, Vol.8, No.3, pp.218-225 (1996)

( 8 ) T. Tsuji, M. Mizoguchi, H. Nishi, and K. Ohnishi: "A velocity measurement method for acceleration control”, Proc. IEEE IECON, pp.1943-1948 (2005)

( 9 ) J.G. López, J. Peleteiro, J.A. Rodriguez, A. Córdova, M.A. González, and J.G. Villa: "Biomechanical Assesment of Aerodynamic Resistance in Proffessional Cyclists: Methodlogical Aspects", Proc. International Symposium on Biomechanics in Sports, pp.286-289 (2002)

(10) K.J. Åström and B. Wittenmark: Adaptive control, Reading, MA: AddisonWesley (2008)

(11) H. Fujimoto and B. Yao: "Multirate Adaptive Robust Control for DiscreteTime Non-Minimum Phase Systems and Application to Linear Motors", IEEE/ASME Trans. Mechatro., Vol.10, No.4, pp.371-377 (2005)

(12) T. Kurosawa, Y. Fujimoto, and T. Tokumaru: "Improvement of Pedaling Torque Estimator for Highly Accurate Pedaling Torque Estimation", Proc. IEE-JIASC, 2-11 (2015) (in Japanese)

Takumi Kurosawa (Student Member) received B.E. and M.E. de-

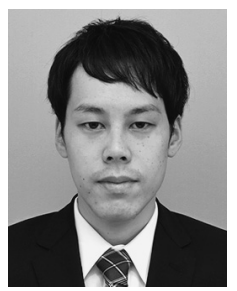
grees in electrical and computer engineering from Yokohama National University, Yokohama, Japan, in 2014 and 2016, respectively. In 2016 he joined IHI corporation, Tokyo, Japan. His research interests include sensorless control of electric power assisted bicycles.

Yasutaka Fujimoto (Senior Member) received B.E., M.E., and Ph.D.

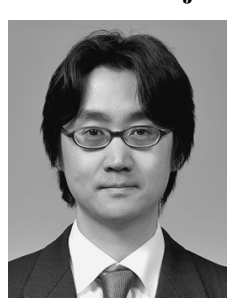
degrees in electrical and computer engineering from Yokohama National University, Yokohama, Japan, in 1993, 1995, and 1998, respectively. In 1998 he joined the Department of Electrical Engineering, Keio University, Yokohama, Japan, as a research associate. Since 1999, he has been with the Department of Electrical and Computer Engineering, Yokohama National University, Japan, where he is currently a professor. His research interests include motion control and actuators, in particular, modeling and control of mobile/legged robots and direct-drive actuators with high-thrust force density and highbackdrivability. He is a senior member of IEEE, and a member of Robotics Society of Japan, SICE, JSAE, IEICE, and INFORMS. 